\title{
Laparoscopic excision of left phaeochromocytoma
}

\author{
KB Galketiya ${ }^{1}$, V Pinto $^{2}$ \\ ${ }^{\text {I}}$ Consultant Surgeon, ${ }^{2}$ Consultant Anaesthetist, Teaching Hospital, Peradeniya. \\ e-mail address of the corresponding author,Dr.K.B. Galketiya: kbgalketiya@yahoo.com
}

\begin{abstract}
Introduction
Phaeochromocytoma is a recognized cause for hypertension. Ninety percent arises in the adrenal medulla. It arises from chromaffin cells and secretes adrenalin and noradrenalin. The common symptoms are headache, palpitations, excessive sweating and associated hypertension. The features may be intermittent or persistent. Surgical excision is indicated ${ }^{1}$.
\end{abstract}

\section{Case report}

A 24 year-old male presented with recurrent attacks of severe headache and palpitations to the medical clinic. Examination of cardiovascular system including measurement of blood pressure was normal. Examination of the nervous system did not reveal any abnormality. However, as the symptoms were severe, it was decided to assess urinary VMA which was found to be elevated. Ultrasound scan followed by a computed tomography revealed a left suprarenal mass of $4.8 \mathrm{~cm} \times 5 \mathrm{~cm}$ consistent with a phaeochromocytoma. He was referred to the surgical clinic. Patient was started on phenoxybenzamine after discussion with the anesthetist.

Laparoscopic excision of the phaeochromocytoma was performed under general anesthesia with endotracheal intubation. The patient was placed in left lateral position. Four ports including the camera port were used. The distal transverse colon, splenic flexure and proximal descending colon were mobilized. The spleen was retracted upwards exposing the suprarenal mass and the left kidney. Upper pole of the kidney was identified after opening the Gerota's fascia. The suprarenal mass was gently dissected initially at its inferior aspect. The adrenal vein was dissected, clipped and divided. The suprarenal mass was dissected using the ultrasonic dissector. During the dissection renal vein and splenic veins were clearly identified. After resection the suprarenal mass was retrieved into a bag (made out of a glove).
Post-operative observation was done under intensive care setting. He was started on feeding and mobilized out the bed on the same day. His blood pressure remained normal. The patient was discharged on the second post-operative day to be followed up at the medical clinic.

\section{Discussion}

Phaeochromocytoma can be successfully managed surgically by an open operation. Various approaches and incisions are described all causing a significant morbidity related to the surgical exposure ${ }^{1}$. Laparoscopic excision is an attractive alternative with significantly reduced morbidity. Both transperitoneal and retroperitoneal laparoscopic approaches have been described ${ }^{2,3}$. There are few contraindications for laparoscopic excision such as mass larger than $10 \mathrm{~cm}$ and suspected malignancy ${ }^{3}$. In the transperitoneal approach as performed in this patient, lateral position is favoured. The adrenal vein has to be ligated at an early stage of the dissection to avoid sudden elevation of blood pressure during manipulation of the tumour. The adrenal arteries can be effectively sealed with the ultrasonic dissector ${ }^{3}$. Once the mass is excised it can be retrieved in a plastic bag. Retrieval bags are commercially available but are expensive. However, a retrieval bag can be made out of a surgical glove. The mass in the bag can be pulled out through a very small incision.

\section{References}

1. Russel RCG, Williams NS, Bulstrode CJK. Parathyroid and Adrenal glands. Bailey \& Love's Short Practice of Surgery. $23^{\text {rd }}$ edition 2000: 745-8.

2. Kirk RM. Adrenal gland. General Surgical Operations 2000: 509-14.

3. Planivelu C. Laparoscopic adrenalectomy: Art of Laparoscopic Surgery ${ }^{\text {st }}$ edition, 2007: 1191-204. 